\title{
Movement-based Sports Video Games: Investigating Motivation and Gaming Experience
}

\author{
Marco Pasch ${ }^{\mathrm{a}, \mathrm{c}}$, Nadia Bianchi-Berthouze ${ }^{*, \mathrm{~b}}$, Betsy van Dijk ${ }^{\mathrm{c}}$, Anton Nijholt ${ }^{\mathrm{c}}$ \\ ${ }^{a}$ Faculty of Informatics, University of Lugano, via Buffi 13, 6900 Lugano, Switzerland \\ ${ }^{b}$ UCL Interaction Centre, University College London, Gower Street, London WC1E 6BT, United Kingdom \\ ${ }^{c}$ Human Media Interaction, University of Twente, PO Box 217, 7500 AE Enschede, The Netherlands
}

\begin{abstract}
Video game consoles that enable gamers to use active body movements are becoming increasingly popular. Yet, little is known about the influence of movement on how gamers experience such games. This study takes an exploratory approach, using different data collection methods. A theory about the relationship between body movement and gaming experience emerges through the systematic collection and analysis of data obtained from interviews, questionnaires, video observations and a motion capture system. A Grounded Theory analysis of the interviews reveals two distinct motivations (to achieve and to relax) with which gamers approach such games, together with two corresponding movement control strategies. Four movement-specific items are found to influence immersion in movement-based interaction: natural control, mimicry of movements, proprioceptive feedback, and physical challenge. These results are verified by exploiting the movement patterns of gamers playing the Nintendo Wii Boxing game. This theory offers insights to game designers as to how to design future generations of movement-based games. Whilst a controller that leaves more space for appropriation can be appealing to a larger population, its design may fail to promote and motivate physical activity and emotional well-being.
\end{abstract}

Key words:

Movement-based Interaction, Exertion, Immersion, Body Movement Patterns, Emotion, Physical Activity, Whole-Body Sports Games, Gaming Experience

\section{Introduction}

Playing video games is often seen as an activity with little to no beneficial outcomes. Together with other sedentary pastimes such as watching television or Internet browsing, playing video games has often been associated with boosting obesity [e.g. 1, 2]. Yet, there is a lack of evidence on effects of video games on gamers, as shown by Lee and Peng [3] in their review of studies into social and psychological effects of computers games. Most studies appear intent on finding negative effects. It is only in the domain of educational games that research has focused on potentially positive outcomes.

A new generation of video games appears to provide for such a positive outcome. Movement-based video games enable their users to employ active body movements as mode of interaction. As such, playing video games could become part of the solution against obesity by requiring their users to be physically active and move away from the couch potato stereotype. Mueller et al. [4] have coined the term exertion interfaces to stress the high amounts of physical activity that such games can elicit. Indeed, movement-based video games can reach activity levels

\footnotetext{
${ }^{*}$ Corresponding author

Email addresses: marco.pasch@usi.ch (Marco Pasch),

n. berthouze@ucl.ac.uk (Nadia Bianchi-Berthouze),

bvdijk@ewi.utwente.nl (Betsy van Dijk), anijholt@ewi.utwente.nl

(Anton Nijholt)
}

that are beneficial for health, and we will discuss studies that measure activity levels in the following section.

When games contribute to a healthier lifestyle, it seems desirable to design them to be as entertaining as possible so as to help ensure they are used frequently. When engaging with movement-based video games is seen as a playful, enjoyable experience (rather than a workout or an exercise), this should help people that usually have a sedentary lifestyle move towards a physically more active lifestyle.

To be able to inform the design of movement-based games, we must first know how gamers appropriate such games (i.e., what movement strategies do they employ), how they experience such games and in particular what influence physical activity has on that experience.

This study aims to contribute to this research endeavour and takes an exploratory approach to investigate the role of body movement in sport video games by using a set of data collection methods. A theory on the relationship between body movement and gaming experience emerges through a systematic collection and analysis of data collected through interviews, video observations and a motion capture system. Gamers are interviewed to investigate what motivates them to engage in movementbased games and how they assess and conceptualize their experiences with those games. A Grounded Theory analysis of these interviews reveals that relaxing and achieving are the two motivations with which gamers approach such games and also 
identifies four movement-specific features that appear to influence immersion in movement-based interaction. These results are provisionally verified by exploring the movement patterns of gamers playing the Nintendo Wii Boxing game. In order to form a theory of the relation between body movement and sport video game experience, we attempt to identify correlations between movement patterns collected through video observations and motion capture, and the findings of the interview study. This theory forms a first step towards a more informed design of movement-based video games.

We start our investigation by presenting a taxonomy of movement-based games and a discussion of potential benefits and risks that arise from them in Section 2. Little is known about how people engage in movement-based interaction. We discuss existing knowledge about motivation for physical activity and experiencing games in Section 3. In Section 4, we present the approach taken in our study and the rationale behind it. Section 5 presents our interview study aiming to identify what motivates gamers to engage in movement-based interaction and how they conceptualize their experiences. The case study on movements of gamers playing Wii Boxing and the relation to the outcome of the interviews is presented in Section 6. We assess our findings and discuss the initial implications of the emerging theory on the design of movement-based video games involving the whole body in Section 7.

\section{Movement-based Interaction}

The best selling video game console at the time of writing this paper is the Nintendo Wii ${ }^{1}$. In a market where competitors have traditionally been aiming to market the console with the highest performance, this statistic seems counter-intuitive. Indeed, out of the so-called "7th-generation" consoles (i.e. Nintendo Wii, Sony Playstation 3, Microsoft Xbox 360), the Wii shows the lowest graphical performance. However, it enables gamers to be physically active in their gameplay and to use active body movements for interaction via handheld controllers.

The central questions of this section are:

- What are movement-based interfaces?

- What are the advantages of such systems?

- What are the risks arising from such systems?

After presenting a taxonomy of movement-based interfaces, we describe potential (health) benefits and potential (health) risks that arise from engaging in movement-based interaction.

\subsection{Game Taxonomy}

Yim and Graham [5] present a two-dimensional taxonomy of games that promote physical activity, based on game world and user interface. Three game worlds are distinguished: Virtual World, Augmented World, and Reality. These are mapped onto

${ }^{1}$ Sanchanta, M.: Nintendo's Wii takes console lead. Financial Times, 12.07.2007. Retrieved from ft.com three interface types: Free Motion Interfaces, Equipment-based Physical Interfaces, and Traditional Electronic Interfaces. Table 1 categorizes existing movement-based games into that taxonomy.

The category Virtual World refers to games that enable an interaction with a virtual world, which corresponds to traditional video games. Yet, to interact with the game, physical movements are necessary. Examples of such systems that have a free motion interface are Nintendo's Wii, Konami's Dance Dance Revolution and Sony's EyeToy. The Wii uses handheld controllers, which are equipped with an infrared camera and accelerometers to locate their position and rotation, and which the user has to wave and swing. The Eye-Toy needs no physical controllers and captures the user's movements with a camera. To play Dance Dance Revolution the user has to jump and dance on a sensor mat.

Examples for systems that combine virtual world with equipment-based physical interfaces are GameBike and PowerGrid Fitness. GameBike is a stationary bike with which racing games on a PlayStation 2 can be steered. An even more specialized example is FlyGuy, a simulator for hang gliders, where gamers are strapped into a frame simulating a hang glider and steer an avatar by twisting and turning their body. Combining Virtual World with what Yim and Graham [5] describe as Traditional Electronic Interfaces leads to what one may call traditional or stationary video games.

Augmented Reality systems combine information from the real world with virtual information. Examples of systems combining augmented reality with free motion interfaces are games such as LaserTag or Human Pacman. LaserTag is a commercially available system in which gamers are equipped with a laser gun and a vest that detects laser 'blasts'. Gamers can run around freely and simulate shooting at each other. Cheok et al. [6] present Human Pacman, where gamers take up the roles of Pacman and the Ghosts and chase each other in real life. Yim and Graham [5] do not provide any examples of augmented reality systems employing equipment-based physical interfaces or traditional electronic interfaces and declare this area as open for research.

As games that take place in reality, Yim and Graham [5] list football as an example of free motion interfaces, cycling as an example of equipment-based physical interface, and radiocontrolled cars as an example of traditional electronic interfaces.

\subsection{Potential Benefits and Risks}

As pointed out earlier, video games are usually seen as a contributing factor to the growing obesity epidemic. Hillier [1] notes that

"children today are engaging much less with the world outside their homes in terms of physical activity ... Technological innovations in media have contributed to these changes, keeping children inside and sedentary in their playtime..." ([1], 56) 


\begin{tabular}{l|lll} 
User interfaces & \multicolumn{3}{|c}{ Game world } \\
& Virtual World & \multicolumn{1}{c}{ Augmented Reality } & Reality \\
\hline Free Motion Interface & Eye Toy & Laser Tag & Football \\
& $\begin{array}{l}\text { Wii Sports } \\
\text { Dance Dance Revolution }\end{array}$ & Human Pacman & \\
\hline Equipment-based Physical Interfaces & $\begin{array}{l}\text { GameBike } \\
\text { FlyGuy }\end{array}$ & Open for Research & Cycling \\
& Traditional video games & Open for Research & Radio Controlled Cars
\end{tabular}

Table 1: Taxonomy of exercise games by game world and user interface (adapted and shortened from [5])

However, the author advocates making technology part of the solution. Video games that promote physical activity can be seen as an example of such a technology-driven solution.

Initial studies show that physical activity during gameplay increases energy expenditure significantly compared to sedentary games. Lanningham-Foster et al. [7] measured the energy expenditure of children playing sedentary video games and playing active video games like Sony's EyeToy and Konami's Dance Dance Revolution. The energy expenditure more than doubles for Dance Dance Revolution and the authors concluded that such games could be useful for obesity prevention and treatment.

Graves et al. [8] also measured the energy expenditure of children playing active video games as compared to sedentary games. Interestingly, they compared the expenditure values of the Wii Sports Bowling and Tennis with those obtained for actual bowling and tennis. The Wii games were shown to require significantly more energy than sedentary activities, but less than the real sports that they simulate. Yet, the measure employed by the authors did not take upper limb movements into account. In a follow-up study [9], the authors used a measure for estimating the energy that included upper limb movements, as indeed those form a crucial part of interacting with the handheld controllers of the Wii. As expected, the authors reported higher activity levels than in the previous study. Figure 1 shows the energy expenditure levels reported for the Wii Sports games, in comparison to a rest value and a sedentary video game on the Microsoft XBOX 360 video game console.

Of all the Wii Sports games (i.e. baseball, bowling, boxing, golf, and tennis), boxing reached the highest activity levels. The authors concluded that while the intensity of real boxing was much higher, the intensity of the Wii Boxing game exceeded the cut-off for moderate intensity physical activity. Thus, it would be high enough to contribute to the recommended amount of daily physical exercise ${ }^{2}$.

Besides obesity prevention, active video games are also envisioned to help with rehabilitation. The field of Virtual Rehabilitation has long used virtual reality technology for rehabilitating patients [e.g. 10, 11]. Recently, video game consoles promoting physical activity have also been deployed in rehabilitation measures. Morrow et al. [12] present a rehabilitation system which is based on Microsoft's XBox. They advocate the use of

\footnotetext{
${ }^{2}$ Lee Graves and Tim Cable, personal communication, 26.03.2008
}

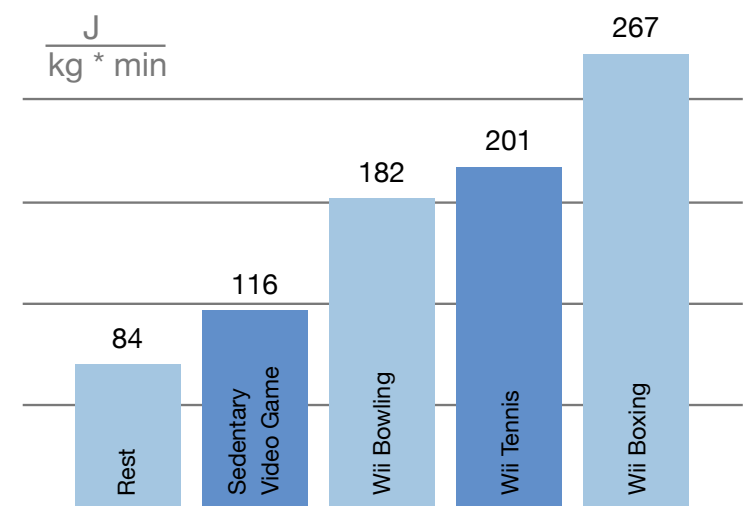

Figure 1: Energy Expenditure of Adolescents whilst Playing Video Games, from Graves et al. [9]

entertainment technology for physical rehabilitation, although mainly to reduce system costs. Galego and Simone [13] combined a Wii remote control and Second Life into a Virtual Rehabilitation system. These authors also stressed the potential of such system as low-cost rehabilitation approaches. Though there are currently no result of scientific evaluations, therapists have already coined the term Wiihabilitation ${ }^{3}$ and report increased motivation in patients who are often unmotivated to carry out very repetitive limb movements.

While game consoles that employ active body movement are used in rehabilitation therapies, they also appear to bring new patients to rehabilitation institutes: Injuries resulting from playing the Nintendo Wii have been reported in popular media and doctors have introduced the condition of Wiitis [14] or Wii shoulder [15]. Bonis [14] describes the condition as follows:

"If a gamer gets too engrossed, he may "play tennis" on the video screen for many hours. Unlike in the real sport, physical strength and endurance are not limiting factors" ([14], 2431)

One can speculate that the reason for such injuries is that gamers do not perceive their video game consoles as sport devices and consequently do not worry about warming up before

\footnotetext{
${ }^{3}$ Tanner, Lindsey (Associated Press). Break a leg? Try "Wiihabilitation". msnbc, 08.02.08. Retrieved on 09.08.08 from http://www.msnbc.msn.com/id/23070190/
} 
playing. This is certainly an issue that should be addressed in future research as well as in future game design.

It is not just physical health that can improve from an increase in physical activity: Riskind and Gotay [16] found that posture influences mental states. Subjects that are put in a hunched and threatened posture report greater stress than subjects that are placed in a relaxed posture. Fox [17] reviewed studies that investigated the influence of physical activity on mental well-being. He concluded that there is growing evidence that exercise increases mental well-being, largely through improved mood and self-perception.

Promoting social interaction is another dimension where physically active games can have beneficial outcomes. For example, Vasilakos et al. [18] presented a distributed theatre, where actors in different places could act together virtually in real-time. It remains to be investigated how the physicality of the interaction improves the interaction compared to a nonphysical setting, or to traditional theatre.

Returning to a video game context, Bianchi-Berthouze et al. [19] found evidence that body movement as an input device not only increases the gamer's level of engagement, it also influences the way the gamer becomes engaged. Their results demonstrate that the controller itself plays a critical role in creating a more complete experience for the gamer.

Whether the increase in engagement in physically active games is due to the actual physical activity or to a higher perceived level of control remains open for research. More knowledge is needed about the link between physical activity and engagement. Thus, it can be concluded that video games that promote physical activity have the potential of improving the gamer's health, however, further investigation is necessary regarding a number of issues. For instance, scientific research can and should assist in solving ergonomic issues, measuring the effectiveness of games, and helping improve the experience of the gamers to increase their motivation to use such systems.

\section{Engaging in Movement-based Interaction}

We have shown that there are many potential advantages to health, as well as a number of risks arising from movementbased interaction. Yet, as we will show in this section, there is only limited scientific knowledge as to how people engage with movement-based interfaces. This section presents the main research questions underlying our investigations:

- What motivates people to engage with movement-based interfaces?

- How do they experience movement-based interaction?

In the following sections, we build towards our research questions by presenting what is known for the respective questions and then discussing their relevance.

\subsection{Motivation}

What motivates people to engage in physical activity? This section gives an overview of theories of enjoyment of physical activity. First, however, it appears reasonable to disentangle

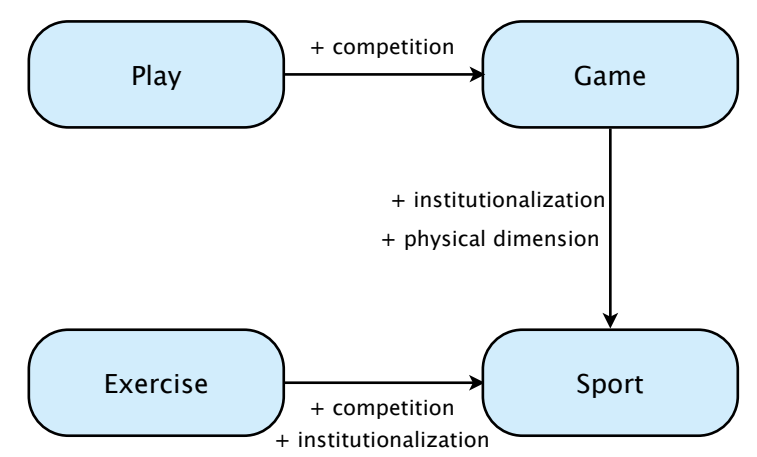

Figure 2: Types of Physical Activities

different types of activity. We discuss play, game, sport, and exercise, before focussing on motivation and enjoyment and clarifying their relationship. Figure 2 illustrates that relationship:

Play can be defined as "behaviour for the purpose of fun and enjoyment with no utilitarian or abstract goal in mind“" ([20], 2). Shaw et al. [20] listed 4 reasons why people play: First, play serves relaxation and recuperative purposes. Second, play can be used to reduce surplus energy. Third, play is an opportunity to practice and rehearse skills. Finally, play can be important to reduce anxiety by confronting one's fears in a safe environment.

Play becomes game when competition is involved in the activity. Shaw et al. [20] define game as "any form of playful competition whose outcome is determined by physical skill, strategy or chance" ([20], 2) and give the following example to illustrate the difference: If one is playing ping pong for fun without keeping score it is play. Once score is kept it is game.

Sport is defined as "institutionalized competitive play involving physical skill, strategy and chance“ ([20], 2). The two criteria that distinguish sport from game are institutionalization and physical dimension. Shaw et al. [20] gave 4 forms of institutionalization: Sport involves a high degree of organization, in terms of governing bodies, leagues, and sponsors. Another form is technological development, which refers to equipment, clothing, and facilities. Ceremonies and rituals add a symbolic dimension to sport. Finally, sport includes educational aspects that are represented by coaches or written manuals. Apart from institutionalization, a physical dimension is required for sport, which does not necessarily require fitness. For instance, dart can still be seen as sport, while bridge hardly qualifies as sport and fits better in the definition of game. Exercise finally is defined as "any form of physical activity carried out for the purpose of health or fitness" ([20], 2).

It should be noted that some activities do not fall into any one category and rather can be seen as hybrids. Still, the framework is helpful to get a clearer view on different types of activities and their specific characteristics.

For instance, are movement-based video games perceived as play, game, exercise or even sport? People that prefer a sedentary lifestyle and do not like to engage in sports and exercise might be discouraged if they perceive movement-based game as sport or exercise. However, seeing it more as playful activity (play), or even competitive but still playful (game), they might 


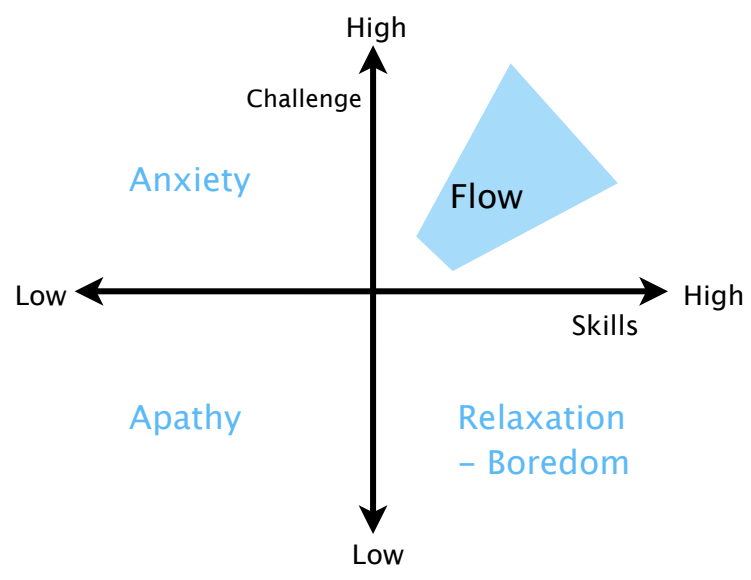

Figure 3: Model of the flow state ([21], 37)

engage with such a game and thus could be persuaded into a more physically active lifestyle.

People that are already sportive might prefer games that explicitly promote exercise or include the framework to be seen as a technology enhanced sport, e.g. with online competitions. So the framework of Shaw et al. [20] can inform game designers on how to design and market their games.

But how do people actually experience sport and exercise? Jackson and Csikszentmihalyi [21] applied flow theory to the sport domain, by relating the components of flow [22] to aspects an athlete should consider in sport. With the limitation of being intended for a broad audience it still gives valuable insights into the study of sport experiences.

Figure 3 shows how flow can only happen when the challenge at hand is matched by a person's skills. When the challenge is too low boredom occurs. If the skills are insufficient, a person might experience anxiety. Both low challenge and low skills result in a state of apathy. It is only when both the challenge is demanding and the skills are high enough to measure up to the task that the state of flow can be reached. In this context, it should be noted that the measurable challenge is not decisive for the experience, but rather it is how a person subjectively estimates the challenge. The same holds for skills: A person might objectively have sufficient skills for a task, but lack of confidence in one's abilities can result in anxiety or apathy to set in.

The consequence for the design of movement-based games is how can the game ensure a balance between challenge and skill. As in strategy games where the artificial intelligence is adapting to the gamer's mental and strategic skills, a movement-based game should adapt to the physical skills of the gamer. Another area of interest is how can game design influence the subjective perception of challenge and skills, and thus the experience of flow. Ultimately, movement based games can succeed only if they offer joyful experiences to the gamer.

\subsection{Gaming Experience}

In the following we present the concept of immersion, one of several concepts used to describe how gamers experience their games. Immersion is a term used widely to describe user experience in HCI, in particular in an entertainment context. The following definition by Murray is quoted widely and has been described as the most accepted [23]:

"The experience of being transported to an elaborately simulated place is pleasurable in itself, regardless of the fantasy content. We refer to this experience as immersion. Immersion is a metaphorical term derived from the physical experience of being submerged in water. We seek the same feeling from a psychologically immersive experience that we do from a plunge in the ocean or swimming pool: the sensation of being surrounded by a completely other reality, as different as water is from air, that takes over all of our attention, our whole perceptual apparatus..." ([24], 98).

Before further investigating the nature of immersion we delineate similar concepts that are all too often used synonymously with immersion. Several authors adapted the aforementioned theory of flow by Csikszentmihalyi [22] to an HCI context: The GameFlow model by Sweetser and Wyeth [25] maps components of flow theory to elements from game design literature. The authors reported that the model in its current state is useful for evaluation of games but needs further development to inform the design of games. An important item of flow theory is the flow zone: A person is in the flow zone when the person's abilities are matched by a challenge. Too much challenge leads to frustration, too little challenge to boredom. Chen [26] recommended that games should adapt to the users " skills in order to keep them in the flow zone.

Presence is another term that appears in the literature to describe the gaming experience. The term originates from studies into virtual reality and is often defined as the feeling "of being there" [27]. Cairns et al. [28] argued that presence in a virtual reality context corresponds to immersion in a gaming context. Similarly, Ermi and Mäyrä [29] preferred the term immersion as "it more clearly connotes the mental processes involved in gameplay“ ([29], 19). We follow this line of argumentation and see immersion as the appropriate term when speaking of user experience in an entertainment context.

In the existing literature on immersion, two models have been proposed that focus on different aspects of immersion. The first model we discuss in the following focuses on the intensity of immersion into a virtual environment, whilst the second distinguishes different types of immersion.

Brown and Cairns [30] investigated the intensity of immersion in video games. From interview data with gamers regarding their experiences during gameplay the authors identified three distinct levels of immersion: engagement, engrossment, and total immersion. For each level, barriers exist that have to be overcome. Figure 4 shows the three levels and their respective barriers.

Engagement is the first level of immersion. To reach it, gamers must be willing to invest time, effort, and attention. In addition, controls and feedback must be provided: "Controls and feedback need to correspond in an appropriate manner so 


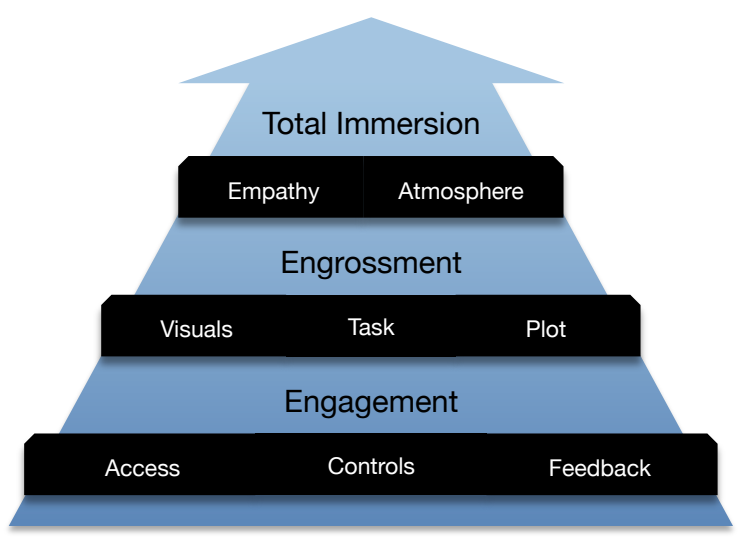

Figure 4: Immersion Model of Brown and Cairns [30], own depiction. Barriers (shown in black) hinder the user from reaching (deeper) immersion.

that the user can become an expert, at least at the main controls" ([30], 1298).

The barrier that has to be overcome to reach the second level, engrossment, is bad game construction, by which the authors referred to visuals, tasks, and plot. They pointed out that at this stage the gamers have already invested emotionally into the game and this makes them continue gaming.

Total immersion is the final level and it is described as being cut off from the world to an extent where the game is all that matters. Barriers to total immersion are a lack of empathy with game characters or a lack of feeling the atmosphere of the game. In a follow-up study, Cheng and Cairns [31] investigated the robustness of immersion. Here, the authors attempted to deliberately break the immersion of their test subjects and found that even low levels of immersion result in subjects ignoring drastic changes in the game's behavior.

Ermi and Mäyrä [29] presented an alternative model of immersion, focussing on different types of immersion, which they derived from interviewing gaming children and their parents. The authors identified three different types of immersion: sensory, challenge-based, and imaginative (SCI), from which they built the SCI-model of immersion, as shown in figure 5. Sensory immersion refers to sensory information during gaming. Large screens and powerful sound are given as examples where sensory information of the real world is overpowered by sensory information from the game world and the gamer entirely focuses on the game. Challenge-based immersion is described as most powerful when a balance between the abilities of the gamer and the challenge of the game is achieved and as such seems to correspond to the flow concept mentioned earlier. Finally, imaginative immersion happens when the gamer gets absorbed with the story line and identifies with the game characters.

Neither of these models contains movement-specific items. Yet we speculate that movement has an influence on immersion for several reasons: First, movement-based interfaces offer a more natural interaction as pointed out in the previous section. This should facilitate the experience of immersion, since users do not have to relay their commands via mouse or keyboard.

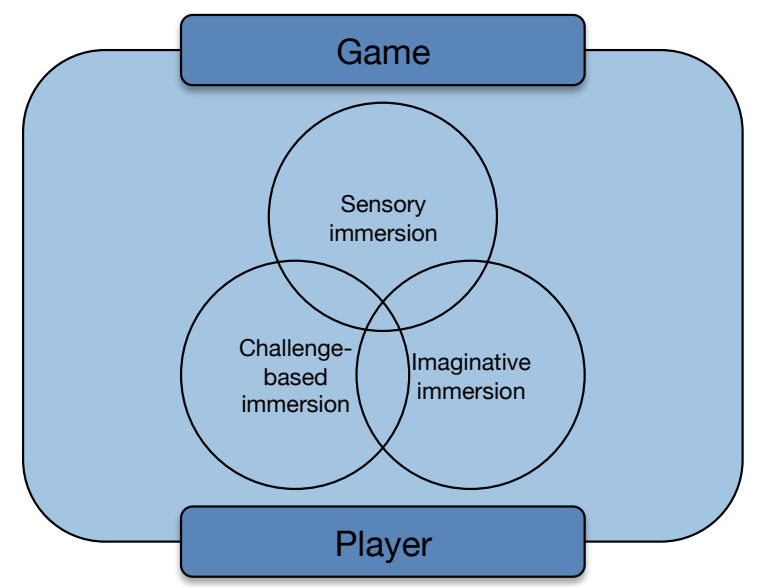

Figure 5: Immersion Model of Ermi and Mäyrä [29], simplified. Three types of immersion are distinguished that can lead the user to immersive states: sensory, challenge-based, and imaginative immersion.

Again, Bianchi-Berthouze et al. [19] found evidence that body movement as an input device not only increases gamers' levels of engagement, it also influences the way gamers become engaged. Body movement appears to help the gamer to enter in the fantasy world and consequently to have a broader emotional experience. That a sense of presence is enhanced by body movement was also shown by Slater et al. [32].

\section{Research Question and Methodological Approach}

In their review of studies into social and psychological effects of video games, Lee and Peng [3] came to the conclusion that there is a lack of research on gameplay as an entertainment experience. They hypothesised that the existing research would be more systematic if it were based on a theoretical understanding of the gaming experience. They concluded that if we really want to understand games, then we need to know how gamers experience their games, a conclusion that was seconded in [29]. Lee and Peng [3] also criticised how most research has been done on media contents, whilst media forms have been neglected. They referred to Reeves and Nass [33] who insisted that

"media forms such as size, fidelity, cuts, synchrony, and movements are equally important factors for determining psychological impact of media" ([3], 340)

Several models have been proposed to account for the gaming experience, the most prominent ones being immersion $[30,29]$ and flow $[25,26]$. But how do gamers experience body movements in their games? The existing scientific models for the gaming experience fail to account for body movements and physical activity during gameplay. Understanding how gamers experience games that enable and require physical activity is a basic requirement if one is to inform designers on how to build games for richer and better experience. Sinclair et al. [34] concluded that "the idea of exergaming is still in its infancy, when it comes to systematic research" ([34], 294). In this paper, we 
ask the question: Is there a relation between gamers' movement patterns during gaming and their gaming experience? In the following subsections, we describe the approach taken to address this question, the game controller chosen for this study, and the rationale behind this choice. Data collection and analysis are reported in the next two sections.

\subsection{Grounded Theory}

Given the limited number of studies performed in this area, our aim is to formulate a theory or a set of hypotheses about the relation between movement and experience that, we hope, will be validated in subsequent studies. Hence, we adopt a grounded theory [35] method to develop a theory from a systematic collection of empirical data. As suggested in [36], these data are collected using different types of method in order to triangulate the findings and provisionally verify the emerging theory. Postgaming experience interviews and questionnaires are used to get the gamers' view on their experience and their body movement strategies. In parallel, video observations and whole-body motion capturing are used for a formal and quantitative analysis of the gamers' movements during game play.

Grounded Theory is a methodology for qualitative analysis, developed by Glaser and Strauss [35]. In Grounded Theory, interview data are analyzed by first assigning codes to statements of the interviewee. The codes are grouped into similar concepts in order to make them easier to analyse and form more abstract categories. These categories form the basis for the creation of a theory, or a reverse engineered hypothesis. This analysis process starts right after the first interview. Preliminary results are then used to further develop the interview guide for the remaining interviews.

Here, we use video observations and motion capture data to verify and further explore the emergent concepts and categories, and thus, to strengthen the validity of the proposed theory. The identified categories and relationships are then further analysed in the context of the literature as another form of validation and understanding.

\subsection{Material}

This study makes use of the Nintendo Wii platform and its sports games. The reason for this choice is twofold. First, and as previously mentioned, the Nintendo Wii was the most popular whole-movement commercial video game at the time of carrying out this study. Second, this gaming console has a very loose control of the movement of the gamer, opening space for gamers to adopt the game in various ways. The Wii Sports game is a collection of five sports simulations: tennis, baseball, bowling, golf and boxing. By using the Wii Remote (also called Wiimote), the gamer can mimic actions performed in real life sports, such as swinging a tennis racket. The rules of each game are simplified to be more accessible to a larger population. The Wiimote is endowed with motion sensing capabilities: it senses the acceleration along three axes and through an optical sensor it senses where it is pointing. Rather than capturing the movement of the body of the gamer, it senses its own movement and as such some movement quality of the gamer. In addition, rather than recognizing the simulated sports movement, it detects the timing of the movement, and the direction in which the Wiimote was moved. For example, gamers in the Wii Boxing game can simulate a punch movement either by extending their arms forward or by simply flicking their wrists forward. Both movements will be considered identical by the game interface because the Wiimote was moved in an identical manner with respect to the sensors of the interface. Thus, this lack of a strict control on the gamers ' body movement enables us to look at different strategies gamers may adopt and what factors may explain such strategies.

\section{Interview Study}

An interview study was conducted to investigate what motivates gamers to play movement-based games and how they experience and interpret their interaction with such systems. Four experienced gamers were enlisted. Experienced gamers were used rather than novices because some level of exposure was required for interviewees to reflect on their experiences with movement-based games.

The following list gives a brief profile of the interviewees:

- i1: female, early 20s, graduate student, experienced gamer, owns a Nintendo Wii, not very sportive

- i2: male, late 20s, employee, little spare time, experienced gamer, has frequently played with the Wii, sportive

- i3: male, early 30s, employee and part time student, little spare time, experienced gamer, owns a Nintendo Wii, rather sportive

- i4: male, mid 20s, graduate student, experienced gamer, has occasionally played with the Wii, sportive, moderately sportive

The interview sessions (20-30 minutes) were held in a semistructured style and initial outcomes were used to update the interview guide for the following interviews. Although we are aware of individual differences in ability to reflect and report about one's own experience, our aim was to identify concepts that can play a role in the relationship between experience and movement rather than compare people"s experience.

The interviews were transcribed and analyzed using the earlier discussed Grounded Theory approach (see subsection 4.1). A 20-minute session of playing the Nintendo Wii Sports games primed the interviewees before the interview took place. The subjects were asked to play two different games on the Nintendo Wii, each for about 10 minutes. The particular games were changed for the different participants to avoid possible biases due to characteristics of a certain game. However, in all sessions it was ensured that participants played one fast-paced game (boxing or tennis) and one slow-paced game (bowling, golf or baseball). This was done as to provide information on how the amount of physical activity, and the type of movement, had affected their gaming experience. The participants were videotaped during game play to provide data regarding certain 
behavioural patterns. These data were also used as evidence when questions arose during the analysis of the interviews.

In the following subsection, we present the outcomes of the interviews. For the sake of providing the reader with a better impression of general trends in the interview data, we provide representative statements where they can help understanding.

\subsection{Motivation and Movement Strategies}

A concept that emerged quite early in the data was that gamers have several distinct motivations to engage with movement-based games. In fact, some experienced gamers seemed to be aware of their changing motivation and adapted their gaming strategy accordingly:

\begin{abstract}
"As you play and play you start to realize that you don't really need to swing and it's just a small movement that you need to make - so I tend to play more technically rather than emotionally. [...] When I am playing to relax and I play baseball, I swing like I would with a real baseball bat. But if I am playing to beat somebody else then I do what I need to do to do the movements." (i3)
\end{abstract}

The statement demonstrates that the gamer is aware he does not need to swing his arm with force. Indeed, the challenge of the game resides in the timing of the movement. Therefore small wrist movements, which allow for a more precise control are often beneficial to achieving a higher score. Nevertheless, the interviewee intimates that he will deliberately make big, forceful movements when his motivation is not to achieve a high score, but just to relax and immerse into the virtual environment. This provides evidence that a gamer can approach the same movement-based game with different motivations.

Two different types of behaviour can also be observed: in the one instance where gamers are playing with the motivation to score points, they employ a strategy that maximises scoring ability. However, when the motivation for playing is to relax by experiencing and/or challenging their movement skills like they would do in a non-competitive sport situation, they employ a different strategy whereby they do the same movements as they would in the actual sport or how they think a good gamer would execute the movement in the real sport. Here, relaxation does not refer to physical relaxation, but rather a mental relaxation that derives from immersing into the game and imagining oneself as playing the actual sport, not just a video game.

Hence, from these interviews two general movement strategies emerge. These strategies appear to be related or derived in the first place from different motivations to play. The first strategy, which we call Game, is characterized by the motivation to achieve. Realistic play appears to be not important and all efforts are pointed towards achieving a high score. The second strategy, which we call Simulation, is employed when gamers want to relax by simulating a real sport. Here, movements are executed in a realistic fashion and mimic the real world equivalent. Scores do not seem to matter.

\subsection{Gaming Experience and Game Realism}

Control appears to be a major factor in the gaming experience that includes body movement and is the first movement feature. It is important for the interviewees to be able to easily understand the game controls. Learning can be facilitated by appealing to the gamer's experience with similar activities in real life. It is seen as positive when gamers can transfer real world knowledge to learn the necessary movements for the game.

"It is like tennis, I really like playing tennis in real life. And with the Wii I really like playing tennis, but you don't have as much as control, you can't move the gamers yourself. So I don't really see it as playing in real life. But then again bowling, it sort of involves the same movements [...] With the bowling you are doing the same as you would be doing in a bowling alley, except for the running. You know, the whole arm chucking movement. Whereas tennis, you're hitting a ball but you don't get that sort of feeling as you would have in real life." (i1)

"The games I liked most so far are the sports games. I don't know why, but the principles are very simple, the controls are very easy and intuitive and it's big fun to play with friends." (i2)

Interviewee 1 describes playing Wii Tennis as an incomplete experience. It does not feel like playing real tennis, whereas Wii Bowling feels authentic. The comments of interviewee 2 seem to suggest that games that mimic real life activities should replicate the movements in those activities quite accurately. They should be "intuitive". For scenarios that mimic real life this is quite straight forward, but it also leads to the question of what determines the movements in a fantasy game with no reference to a real world scenario.

Another important concept can be described as mapping of movements. This refers to how well the gamer's movements are replicated on screen and how the game reduces the high number of degrees of freedom in the movements a gamer can make, as exemplified by the following comments:

"... But I think with the technology that we have so far it might be limited how it can be really reflected. In boxing for example, what I said earlier, the type of punches that I can do are not really reflecting the diversity that I can have in real life.“ (i2)

Interviewee 2 states that he is unsatisfied with the fact that the system does not replicate the movements exactly as he executes them. Still, he acknowledges that there are technical limitations involved. Interestingly, when it comes to the Wii Tennis game, interviewees are positive about the fact that they cannot steer the movements of the avatar itself, but only execute the swings, stating that this alone is difficult enough.

"My movements were a bit larger and faster than the ones that the avatar was making. And sometimes the 
avatar was loosing its balance or something. It was leaning in one direction and I was in another and it was taking a while to catch up with me."(i4)

Feedback is another recurring concept in the data, though the term feedback itself is not mentioned explicitly by the interviewees. We interpret the statements in a way that the body itself is a source of feedback for the user. In the example above, interviewee 4 describes that he is physically leaning in one direction, whereas the avatar is still leaning in another direction. This discrepancy is related to the feature "mapping of movements" that was described above, but it also shows that the positioning of the body is a source of feedback that, in the game platform used in our study, is not in agreement with the visual feedback coming from the screen. Through the movements, the gamer receives additional feedback in the form of proprioceptive feedback. In traditional video games sensory immersion is limited to sight, hearing and touch. These senses belong to the so-called exteroceptive senses (i.e. hearing, sight, smell, taste, and touch). The proprioceptive sense provides information about the relative position of neighboring parts of the body.

Challenge in movement-based interaction appears not only to have a mental, but also a physical component. The fourth feature that we can identify from the interview data is physical challenge. The interviewees frequently stated feeling physically challenged by the game and being exhausted afterwards.

\subsection{Discussion}

We discuss the results of our interview study by applying the four movement features that emerged to the two aforementioned models of immersion. The features and the respective model constituents are shown in Figure 6. We then discuss each relation and how the respective feature can potentially influence immersion.

Movement-based interfaces provide for a more natural form of control than mouse, keyboard or joystick. Using body movement is often described as intuitive by the interviewees and also critized when it is not resembling movements in real life as expected. In the immersion model of Brown and Cairns [30], control is a barrier to the first level of immersion. A more natural mode of control can lower this barrier and facilitate immersion, given that the interaction resembles movements in real life.

Mimicry of movements is the second movement feature identified from interview data. When the avatar copies the movements of the gamer, this appears to raise the level of empathy that is felt with the avatar, as exemplified by the following quotes:

"The boxing also felt more personal, because it feels like someone is hitting back at you, although that's not the case. So it's more emotionally engaging." (i3)

"Keeping your arms up all the time and trying eagerly to punch and being in a situation where you can virtually be punched as well is maybe more stress than bowling." (i2)
Interviewee 3 reports about the Wii Boxing game that it "feels like someone is hitting back at you." Although he immediately reflects that this is not possible, there seems to be a strong emotional connection to the avatar. The same holds for interviewee 2 who finds a situation where he can be hit, if only virtually, stressful. As shown by Bailenson and Yee [37], mimicry can lead to increased empathy with a virtual character. In the case of movement-based video games like Wii Boxing, the avatar copies the movements of a gamer. It is conceivable that this mimicry of the gamer's movements leads to a stronger identification with the avatar. Empathy is also a barrier to immersion and mimicry of movements appears to have the potential to lower this barrier. It also seems to facilitate imaginative immersion.

Proprioceptive feedback is the third movement feature. With regards to the barriers to immersion this additional channel of immersion offers the potential of lowering the barrier feedback. When we look at the other model of immersion, it appears to facilitate sensory immersion. Furthermore, as discussed in the literature review, proprioceptive feedback can affect emotional experience [16].

Physical challenge is the final movement feature. In static games, the challenge is usually placed on the gamer's mental capabilities. Racing games and first-person shooter games could be considered an exception as they also challenge the gamer's reflexes, and indeed, they could be categorised as movement-based games. In movement-based interaction, the physical challenge offers an additional channel of feeling challenged.

\section{Case Study: Movement Patterns in Wii Boxing}

As a form of preliminary validation of the categories and their relationships identified in the previous section, and to further identify other factors that could have an impact on gamer experience, a second study was run to observe and record the movement of gamers of the boxing Wii game.

For this experiment, participants were enlisted from graduate students at UCL. They were recruited via personal communication with no incentives given. After consenting to take part and filling in an initial questionnaire, participants were fitted with an inertial gyroscopic motion capture suit (Gypsy 6, Animazoo, Brighton, UK). They were then given a short introduction to the Wii Boxing game and instructed to play for 15 minutes. To avoid biasing the participants, the experimenter left the room during the gaming session. After 15 minutes, the experimenter returned and the participants were released from the motion capture suit. They were then asked to fill in a second questionnaire.

The experiments were held in a room with enough space on all sides to move freely during game play. The output of the Wii was projected onto a screen with a diameter of approximately 2 meters. The Wii Boxing game was chosen, as it is the game with the highest activity levels within the Wii Sports games. 


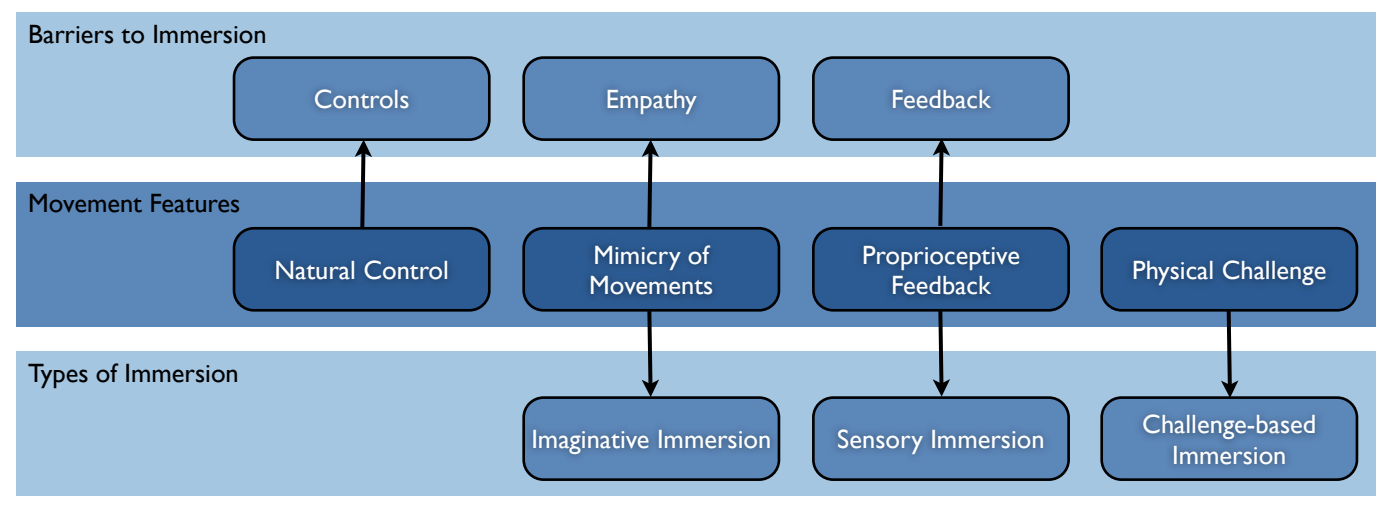

Figure 6: Movement features identified and their potential influence on barriers to immersion [30] and types of immersion [29]

\subsection{Questionnaires}

In addition to validating the previous outcomes, questionnaires were used to identify factors that could influence movement patterns. The approach at this point is still an exploratory one and there are assumptions rather than strong hypotheses behind the selection of the questionnaires. Here, we provide a brief explanation of the questionnaires chosen and the motivation for choosing those.

Before the gaming session, participants were asked to fill in the following questionnaires:

- Big Five Inventory [38], 44 items

- Sports Motivation Scale [39], 28 items

- Leisure Motivation Scale[40], 28 items

- Prior Experience with Wii and Boxing (own compilation), 9 items

The first questionnaire given before the gaming session is the Big Five Inventory [38], a questionnaire assessing personality that consists of 44 items and scoring participants on five personal traits (i.e., Extraversion, Agreeableness, Conscientiousness, Neuroticism and Openness). The interview study revealed motivation as a decisive factor for the way a gamer engages with a movement-based game. The personality questionnaire was employed to assess whether personal traits could be correlated to motivation and movement patterns. For example, it is conceivable that an extrovert person could exhibit larger movements than an introvert person. The Sports Motivation Scale [39] and Leisure Motivation Scale [40] were chosen to further investigate the phenomenon of motivation and how it influences movement patterns. Both scales consist of 28 items that score participants on seven sub-scales: three types of Intrinsic Motivation (i.e. engaging in an activity purely for the pleasure of it), three types of Extrinsic Motivation (i.e. engaging in an activity for reasons outside the activity, a means to an end), and Amotivation (i.e. motivation is lost or there never was motivation for an activity).

The idea behind using the Sports Motivation Scale and Leisure Motivation Scale was to investigate whether motivation-related issues have an influence on the way a gamer moves. For example, it is conceivable that a person that likes accomplishing things could be more physically active in order to achieve victory in a movement-based game.

An additional, self-compiled questionnaire was given, that asked about participants' prior experience with the Nintendo Wii in general, the Wii Boxing game in particular, and also their prior experience with (and knowledge of) boxing and martial arts. The assumption was that prior experience e.g. with boxing could impact the movement pattern of a gamer. Similarly, a gamer with a lot of experience on the Wii might have developed a movement pattern different from that of a novice.

After the gaming session, participants were again asked to fill in questionnaires:

- Physicality and Wii specific items (own compilation), 9 items

- Rating of Perceived Exertion [41], 2 items

- Self-assessment Manikin [42], 3 items

- Immersion Questionnaire (Jennett et al., unpublished), 31 items

The first questionnaire after the gaming session asked participants about their view on statements about the gamer's experience and movements. The Rate of Physical Exertion (RPE) is a measure for self-reported exertion, where athletes, for example, rate the exertion they experience on a scale from 6 to 15 [41]. At several points on the scale, activities with a comparable level of exertion are given as a reference, for example, walking slowly at one's own pace for some minutes corresponds to a rating of 9. Here, the RPE scale was used to check if the experience of the gamers influenced their perception of exertion.

The Self-Assessment Manikin [42] is a measure for selfreported levels of one's emotional state. It shows a rather abstract figure in several instances depicting varying levels of valence, arousal, and dominance. Here, the manikin was used as a measure of how gamers rated their own emotional states during gaming and to investigate if there was a connection to their movements. 

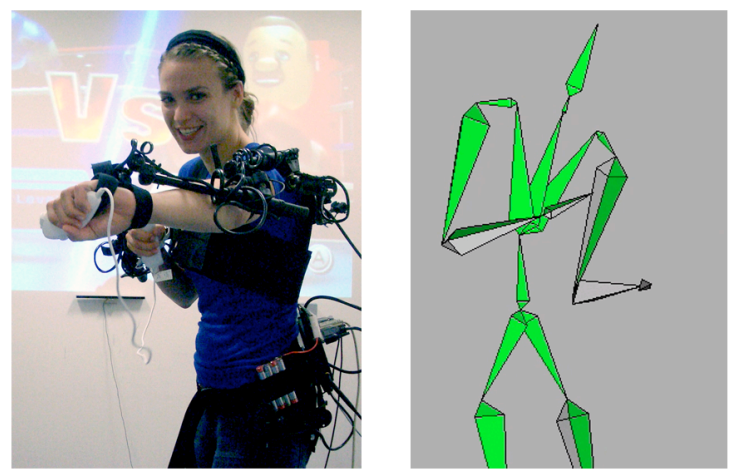

Figure 7: Gypsy Motion Capture System; Left: Participant in Gypsy 6 Motion Capture Suit (image shown with written consent); Right: Output Motion Capture Data on Human Model

\subsection{Motion Capture, Video Recordings, and Observer Ratings}

The Gypsy 6 (Animazoo, Brighton, UK) motion capture suit is an inertial gyroscopic motion capture suit. It is an exoskeleton that is equipped with 37 potentiometers and 2 gyros that measure the rotations around 16 joints, on both upper and lower body. In addition, the position of the suit in 3D space is measured. The sampling rate of the suit is $120 \mathrm{~Hz}$. The data is sent via USB cable or wireless to a PC where the movements can be observed in real-time and are saved in BVH (Biovision Hierarchy) files. Figure 7 shows a participant wearing the suit and the output of the motion capture data on a model of the human body.

In addition to the motion capture data, video recordings were made from a frontal-lateral angle and from over the shoulder of the participant, to be able to correlate movements that participants made to game events. Also, this allowed to assess the performance of the participant, as shown in Figure 8 and discussed below.

To get an alternative measure to the self-report via questionnaires, the recordings from the frontal-lateral angle were also used to let observers rate the movements of the participants. The concrete question was: "Does it look like the gamer is really boxing somebody?" This question had to be answered on a scale from 1 (total disagreement) to 5 (total agreement). Five observers were asked to rate video clips of the participants. The observers were recruited from students at UCL.

Each observer individually rated 40 video clips, the first 10 being training data that was not used for analysis. The remaining 30 clips consisted of 3 clips of each of the original 10 participants, showing them at different points into their gaming session. The clips were shown in a random order. Each clip had a length of 10 seconds and the average rating session took about 20 minutes. 10 seconds appeared as a good compromise between the clips being long enough to allow observers to get a good impression of the participants and short enough to keep the total session time short enough to not tire observers overly. The inter-rater agreement between observers showed a very high level of concordance with a Cronbach alpha of 0.81 .

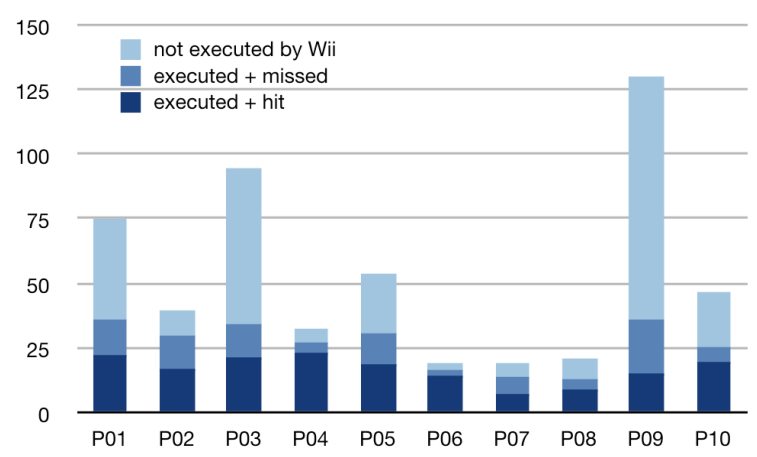

Figure 8: Video Annotation: Punches over a period of 20 seconds

\subsection{Analysis}

Although the original aim was to have 20 participants to allow a statistical analysis, the number of participants was reduced to 10 (thereof 7 males; mean age: 26 yrs, SD: 2.6) due to mechanical failures resulting from stress imposed on the exoskeleton by the powerful punches of some participants.

A first analysis step was the visual inspection of the video footage. This revealed great differences in playing styles. Some participants showed extensive arm movements, whereas others only made small arm movements. Differences were observed for punch frequency, punch amplitude, and overall body movement. To quantify the features deemed important during the visual inspection, short segments of the video recordings were annotated. In the Wii Boxing game, a gamer advances through a series of short fights against increasingly stronger opponents. Each segment was taken from the beginning of a new round of boxing towards the middle of the gaming session. The length of each segment was 20 seconds. It was not possible to analyze a longer segment as knock-downs and other game events did not allow longer segments of continuous boxing activity.

A measure that appeared to be important was punch frequency. Figure 8 shows a representation of the punch frequency distinguishing between when a gamer punches but the Wii does not execute the punch in the game, when a gamer punches but misses the opponent ("executed + missed"), and when a gamer punches and hits the opponent ("executed + hit"). The Wii does not execute a punch when a punch is too soft or when a gamer punches while the avatar is still in the process of executing a previous punch or is recovering from being hit. The respective values are given in Table 2.

The numerical data of the motion capture suit was inspected to reveal more features that can be used to characterize the different playing styles. First, the amount of total movement of the gamers was calculated. It is defined as the accumulation of displacement of the body core, again over a period of 20 seconds. Another measure that emerged during visual inspection was the angular displacement of the elbows. This feature is defined as the accumulation of the angular displacement (in arc degrees) of both elbows combined over a period of 20 seconds. The results are given in Table 3. Finally, the average punch amplitude for each participant was measured. Table 3 shows the average punch amplitude for the X-Rotation (flexion/extension) of the 


\begin{tabular}{|c|c|c|c|c|c|c|c|c|c|c|}
\hline & P01 & P02 & P03 & $\mathrm{P} 04$ & P05 & P06 & P07 & P08 & P09 & P10 \\
\hline Number of Punches & 74 & 39 & 93 & 31 & 53 & 19 & 18 & 20 & 129 & 46 \\
\hline Ratio Hit to Total Punches & .28 & .41 & .22 & .71 & .34 & .68 & .33 & .40 & .11 & .41 \\
\hline Ratio Executed to Total Punches & .47 & .74 & .35 & .84 & .57 & .84 & .72 & .06 & .27 & .54 \\
\hline
\end{tabular}

Table 3: Motion Capture Data: Angular Displacements of Elbows (Accumulated over 20 Seconds) and Average Punch Amplitude (X-Rotation, i.e. flexion/extension)

\begin{tabular}{|c|c|c|c|c|c|c|c|c|c|c|}
\hline & P01 & P02 & P03 & P04 & P05 & P06 & P07 & P08 & P09 & P10 \\
\hline Angular Displacements of Elbows & 55637 & 6539 & 47717 & 37274 & 35786 & 9264 & 14516 & 8190 & 13296 & 15699 \\
\hline Average Punch Amplitude & 95 & 8 & 118 & 160 & 90 & 63 & 90 & 105 & 18 & 75 \\
\hline
\end{tabular}

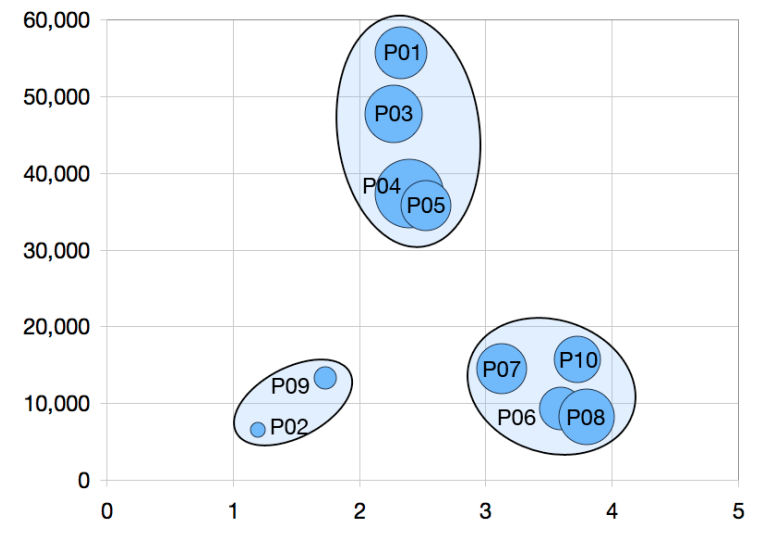

Figure 9: Clustering: Observer Ratings of Boxing Realism (X-Axis; Scale 1-5) vs. Angular Displacement of the Elbows (Y-Axis; accumulated over 20 seconds) vs. Average Punch Amplitude (Bubble Size; Size of X-Rotation, i.e. rotation in forward direction)

elbows.

\subsection{Results}

The quantified movement features, questionnaire results, and observer ratings were investigated for correlations. Again, this was done visually as a total of only 10 data sets did not allow for a proper statistical analysis.

When plotting the observers ' ratings of whether they thought the gamers were really boxing, against the angular displacement of the elbows, three clusters that correspond to the playing styles mentioned above can be identified (see Figure 9, which also indicates the average punch amplitude as bubble size).

The first cluster (P02, P09) only gets a low realism rating and is further characterized by low amounts of angular displacements and punch amplitudes. The second cluster (P01, P03, $\mathrm{P} 04, \mathrm{P} 05)$ receives medium realism ratings, and displays high angular displacements levels and big punch amplitudes. The group with the highest realism ratings (P06, P07, P08, P10) only shows low amounts of angular displacements. Yet, the size of the bubbles reveals that they show medium to large punch amplitudes. The video footage indeed reveal that these gamers reacted to events that happened in the game, i.e. they waited for a good moment to punch the opponent and they also took a defensive stance while waiting.

The results from the questionnaires were compared to the clusters to check for correlations with personality traits and motivation but none could be found. This could be due to the small data sample and further investigation will be necessary to assess whether personality plays a role in the preferred strategy patterns. Nevertheless, interesting observations were made regarding the movement strategies used by the participants in relation to the results of the interview study.

The interview study revealed that gamers approach the Wii Sports games with two motivations (Achieving and Relaxing) and with two corresponding strategies which were identified as Game and Simulation. Out of the three patterns identified by the movement study, one pattern (the lower right cluster in Figure 9) corresponds to the strategy Simulation: it can be described as the involvement of the whole body, where movements are executed according to the role play of the game. The pattern Simulation is characterized by gamers that observed the action on the screen and reacted to it. They had a lower number of punches as they waited their turn and did not punch blindly. When they did punch, however, they did so with large arm extensions, as is done in real boxing, which they simulated. The result is a lower value for the angular displacement of the elbows. Their defending movements consisted in raising the arms to protect the face and in laterally bending the trunk and ducking. Their posturing and arm extension during punches seemed as though they were really trying to reach their opponent.

For the strategy Game, the pattern corresponds to the lower left cluster in Figure 9 and can be described as a low involvement of the body. Only movements that are absolutely necessary to interact with the game are executed. For the Wii Boxing game this means that the body core remains stationary and there are only very small arm extensions. Yet, the gamers that exhibited this pattern showed a high punch frequency. These gamers had learned that for the Nintendo Wii the punch amplitude is irrelevant and that a short impulse is enough to perform a punch. The high punch frequency led to a good performance in terms of total hits, even though many punches did not hit or were not executed at all. Still, the level of physical activity remained low and the video recordings showed they did not appear to be emotionally engaged.

An intermediate pattern is captured by the upper cluster in Figure 9 and can be described as the involvement of the whole body with little or low resemblance to boxing. The gamers showed large extensions of the arms whilst also punching at a high frequency, to the extent that it appeared they were over pacing themselves. Their behaviour often seemed to be independent of game events, i.e. they showed only little defensive behaviour, even when their avatar was hit repeatedly. 
Some of the gamers in this group showed various exploratory movements probably aimed at understanding how to control the game.

\section{Discussion and Conclusions}

The research presented in this paper aims to provide a better understanding of how body movement may affect gamers' experiences in movement video games, which involve the whole body. As a case study we focused on the sport game Wii Boxing. Through interviews, observations and measurement of body movements of gamers, a model of the relationship between gamer motivation and movement patterns during gaming emerged. Figure 10 gives an overview of this relationship.

When gamers play to relax, they simulate the sports" movements. Their bodies are completely involved in the game. Even though movements are not necessary, the gamers ' main pleasure derives from acting the movements rather than from winning the game. What we call relaxing seems to correspond to what Shaw et al. [20] call play: a behaviour for the purpose of fun and enjoyment with no utilitarian or abstract goal in mind. Play serves relaxation and recuperative purposes, reduces surplus energy, is an opportunity to practice and rehearse skills, and can be important to reduce anxiety by confronting one's fears in a safe environment. Interviewees mentioned relaxation throughout the interviews, not in a physical sense but from a mental viewpoint. They achieved this by playing the games in a realistic fashion, immersing into the virtual world. Staying in the context of the Wii Sports games, they mimicked the movements of what they thought a professional athlete would do.

When achieving is the motivation, gamers are less concerned with executing realistic movements. Here, movements are reduced to what is necessary. The movement patterns that can be observed are the ones that, to the knowledge and skill of the gamers, can lead to high scores. For a skilled gamer, the movements are reduced to the necessary minimum, possibly losing any resemblance to the movements of the sports, which the game should simulate unless required by both controller and game interface. In the case of the Wii, experienced gamers have learned that small, jerky movements are sufficient and even more efficient for control than large movements.

In the case of poor skills, explorative behaviour can be observed. This behaviour aims for understanding how the controllers work and how body movements can be used to control the game. The motivation of the gamers as well as the design of the game may have an effect on biasing this exploration versus the controller or versus the body movement.

We also identified four movement-specific features that appear to influence immersion into movement-based interfaces. Movement-based interfaces can give gamers a higher feeling of control, as the interaction is more direct and resembles movements that have been learned from real life (if implemented well). When the avatar on screen mimics the movement of the gamers, this also appears to raise the level of empathy felt for the avatar and facilitates immersion into the virtual. Our built-in ability of sensing the position of our body parts even when we cannot see them (proprioception) is activated when engaged in movement-based interaction and gives us an additional channel of feedback as compared to traditional static games. Finally, challenge experienced in movement-based games is not only mental. A second dimension arises in the form of feeling physical challenge.

Although further studies with a larger population are necessary to validate this theory, this relationship is of great importance to designers. Designing to foster movement rather than typical game behaviour requires that the controller and the interface of the game be designed to require specific movements and to give proper feedback about the movement being performed. Proper feedback is necessary not to disorient gamers. The interview study showed that a perfect similarity between the game and the sport being simulated is not always necessary. The complexity of the movement necessary to play the game should be sufficient to enable gamers to feel that they are simulating the sport but not too complex as to create a barrier against becoming immersed in the game, as also suggested in [31]. As the skills of gamers increase, more complex movements may become necessary to trigger a sense of role play in them.

Although one could design games for a chosen market (e.g., either achievement-based games or fitness games), personalization mechanisms could be embedded to fit the gamers ' motivation. Whilst a completely automatic analysis of the gamers" movement style may be very challenging, we have shown that some features could be used as indicators of their motivation. The average punch amplitude is a good indicator for distinguishing between low-intensity patterns of Game and highintensity patterns of Simulation. The angular displacement of the elbows provides the means to distinguish between the highintensity pattern of Game and Simulation. In principle, both the average punch amplitude and the angular displacement of the elbows should be easily obtainable from the Wii controllers as they are equipped with accelerometers. Alternatively, an additional sensor attached to the arms could deliver the required information. In other words, it should be quite easy to observe patterns and from this to deduce the strategy and motivation of the gamers. Further analysis of the motion capture data should lead to identify other interesting features that have been singled out by visual inspection of the videos. In particular, the defensive behaviour offered clear distinction between the three different groups.

In conclusion, while the specific movement patterns we observed in the movement analysis study are specific to the Wii Boxing game, the outcomes of the interview study are valid for movement-based sport video games in general, since they are based on a general discussion of sport games making the proposed model (Figure 10) generalizable to other sports games. Furthermore, there is evidence from other studies that this model could also be valid for other types of movement-based video games. For example, Bianchi-Berthouze et al. [19] found evidence that realistic body movement as an input device in music games not only increases gamers' levels of engagement, but it also influences the way gamers become engaged. Body movement appears to help the gamer to enter in the fantasy world and consequently to have a broader emotional experience. Lindley et al. [43] have also shown that, by affording realistic move- 


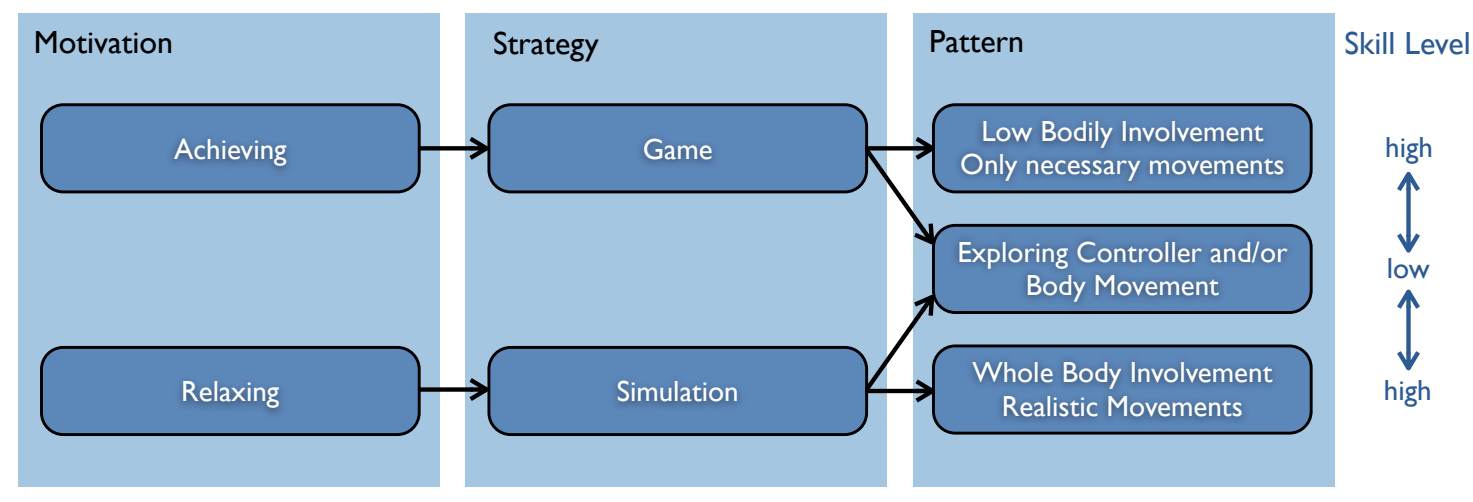

Figure 10: Motivations, Strategies, and Movement Paterns for Nintendo Wii Boxing

ments (Simulation) in music games, pairs of gamers became more socially interactive than when they play without realistic movements (Game).

We need however to acknowledge that our results are based on a limited number of participants. Research drawing from a larger population is needed to further validate the outcomes from our exploratory interview and movement analysis studies.

\section{Acknowledgements}

Marco Pasch wishes to acknowledge support from the Twente Mobility Fund (TMF). The research of Nadia BianchiBerthouze has been supported by the Marie Curie International Re-Integration Grant "AffectME" (MIRG-CT-2006-046343). The research of Betsy van Dijk and Anton Nijholt has been supported by the GATE project, funded by the Netherlands Organization for Scientific Research and the Netherlands ICT Research and Innovation Authority (ICT Regie).

\section{References}

[1] A. Hillier, Childhood overweight and the built environment: making technology part of the solution rather than part of the problem, The ANNALS of the American Academy of Political and Social Science 615 (1) (2008) $56-82$.

[2] L. Epstein, J. Roemmich, J. Robinson, R. Paluch, D. Winiewicz, J. Fuerch, A randomized trial of the effects of reducing television viewing and computer use on body mass index in young children, Archives of Pediatrics and Adolescent Medicine 162 (3) (2008) 239 - 245.

[3] K. M. Lee, W. Peng, What do we know about social and psychological effects of computer games? a comprehensive review of the current literature, in: P. Vorderer, J. Bryant (Eds.), Playing Video Games: Motives, Responses, and Consequences, Lawrence Erlbaum Associates, Mahwah, N.J., 2006, pp. $259-274$.

[4] F. Mueller, S. Agamanolis, R. Picard, Exertion interfaces: sports over a distance for social bonding and fun, in: $\mathrm{CHI}$ '03: Proceedings of the SIGCHI conference on Human factors in computing systems, New York, NY, 2003, pp. 561-568. doi:10.1145/642611.642709. URL http://dx.doi.org/10.1145/642611.642709

[5] J. Yim, N. T. C. Graham, Using games to increase exercise motivation, in: Future Play '07: Proceedings of the 2007 conference on Future Play, 2007, pp. 166-173. doi:10.1145/1328202.1328232. URL http://dx.doi .org/10.1145/1328202.1328232

[6] A. D. Cheok, K. Goh, W. Liu, F. Farbiz, S. Fong, S. Teo, Y. Li, X. Yang, Human pacman: a mobile, wide-area entertainment system based on physical, social, and ubiquitous computing, Personal and Ubiquitous Computing 8 (2) (2004) 71-81. doi:10.1007/s00779-004-0267-X. URL http: //dx.doi .org/10.1007/s00779-004-0267-x
[7] L. Lanningham-Foster, T. B. Jensen, R. C. Foster, A. B. Redmond, B. A. Walker, D. Heinz, J. A. Levine, Energy expenditure of sedentary screen time compared with active screen time for children., Pediatrics 118 (6) (2006) 1831-1835. doi:10.1542/peds.2006-1087.

URL http: //dx.doi .org/10.1542/peds . 2006-1087

[8] L. Graves, G. Stratton, N. D. Ridgers, N. T. Cable, Comparison of energy expenditure in adolescents when playing new generation and sedentary computer games: cross sectional study, BMJ 335 (7633) (2007) 12821284. doi:10.1136/bmj.39415.632951.80.

URL http://dx.doi.org/10.1136/bmj.39415.632951.80

[9] L. E. Graves, N. D. Ridgers, G. Stratton, The contribution of upper limb and total body movement to adolescents' energy expenditure whilst playing nintendo wii, European journal of applied physiology 104 (4) (2008) 617-623. doi:10.1007/s00421-008-0813-8.

URL http: //dx .doi .org/10.1007/s00421-008-0813-8

[10] G. Burdea, Virtual rehabilitation - benefits and challenges, Methods Inf Med 42 (5) (2003) $519-523$.

[11] M. K. Holden, Virtual environments for motor rehabilitation: review, Cyberpsychology \& behavior : the impact of the Internet, multimedia and virtual reality on behavior and society 8 (3). doi:10.1089/cpb.2005.8.187. URL http: //dx . doi .org/10.1089/cpb. 2005.8.187

[12] K. Morrow, C. Docan, G. Burdea, A. Merians, Low-cost virtual rehabilitation of the hand for patients post-stroke, in: International Workshop on Virtual Rehabilitation, 2006, pp. 6-10.

[13] B. Galego, L. Simone, Leveraging online virtual worlds for upper extremity rehabilitation, in: IEEE 33rd Annual Northeast Bioengineering Conference (NEBC '07), 2007, pp. $267-268$.

[14] J. Bonis, Acute wiiitis, N Engl J Med 356 (23) (2007) 2431-2432. doi:10.1056/NEJMc070670. URL http://dx.doi.org/10.1056/NEJMc070670

[15] A. Cowley, G. Minnaar, Watch out for wii shoulder, British Medical Journal 336.

[16] J. H. Riskind, C. C. Gotay, Physical posture: Could it have regulatory or feedback effects on motivation and emotion?, Motivation and Emotion 6 (3) (1982) 273-298. doi:10.1007/BF00992249. URL http://dx.doi.org/10.1007/BF00992249

[17] K. Fox, The influence of physical activity on mental well-being, Public Health Nutrition 2 (3a) (1999) $411-418$.

[18] A. V. Vasilakos, W. Liu, T. H. D. Nguyen, T. C. T. Qui, L. C. Chen, C. B. Tovar, D. J. Díaz, A. D. Cheok, G. Marentakis, Interactive theatre via mixed reality and ambient intelligence, Inf. Sci. 178 (3) (2008) 679-693.

[19] N. Bianchi-Berthouze, W. Kim, D. Patel, Does body movement engage you more in digital game play? and why?, in: A. Paiva, R. Prada, R. W. Picard (Eds.), Affective Computing and Intelligent Interaction, 2007, pp. 102-113. doi:10.1007/978-3-540-74889-2_10.

URL http://dx.doi .org/10.1007/978-3-540-74889-2_10

[20] D. Shaw, T. Gorely, R. Corban, Sport and Exercise Psychology, BIOS Scientific Publishers, 2005.

[21] S. A. Jackson, M. Csikszentmihalyi, Flow in sports: the keys to optimal experiences and performances, Human Kinetics, 1999.

[22] M. Csikszentmihalyi, Flow: The Psychology of Optimal Experience (P.S.), Harper Perennial, 1990. 
[23] A. McMahan, Immersion, engagement and presence, in: M. Wolf, B. Perron (Eds.), The Video Game Theory Reader, Routledge, 2003, pp. 67 86.

[24] J. Murray, Hamlet on the Holodeck: The Future of Narrative in Cyberspace, MIT Press, Cambridge, 1997.

[25] P. Sweetser, P. Wyeth, Gameflow: a model for evaluating player enjoyment in games, Computers in Entertainment 3 (3) (2005) 1 - 24.

[26] J. Chen, Flow in games (and everything else), Commun. ACM 50 (4) (2007) 31-34. doi:10.1145/1232743.1232769.

URL http://dx.doi.org/10.1145/1232743.1232769

[27] W. Ijsselsteijn, G. Riva, Being there: the experience of presence in mediated environments, in: G. Riva, F. Davide, W. Ijsselsteijn (Eds.), Being There: Concepts, Effects and Measurements of User Presence in Synthetic Environments, IOS Press, Amsterdam, 2003, pp. 3 - 16.

[28] P. Cairns, A. Cox, N. Berthouze, S. Dhoparee, C. Jennett, Quantifying the experience of immersion in games, in: Cognitive Science, Vancouver, 2006.

[29] L. Ermi, F. Mäyrä, Fundamental components of the gameplay experience: analysing immersion, in: S. de Castell, J. Jenson (Eds.), Changing views: worlds in play, selected papers DiGRA conference, 2005, pp. 15 - 27

[30] E. Brown, P. Cairns, A grounded investigation of game immersion, in CHI '04: CHI '04 extended abstracts on Human factors in computing systems, ACM Press, New York, NY, USA, 2004, pp. 1297-1300. doi:10.1145/985921.986048. URL http://dx.doi.org/10.1145/985921.986048

[31] K. Cheng, P. A. Cairns, Behaviour, realism and immersion in games, in: CHI '05: CHI '05 extended abstracts on Human factors in computing systems, ACM, New York, NY, USA, 2005, pp. 1272-1275. doi:10.1145/1056808.1056894. URL http://dx.doi .org/10.1145/1056808.1056894

[32] M. Slater, A. Steed, J. McCarthy, F. Marinelli, The influence of body movement on presence in virtual environments, Human Factors: The Journal of the Human Factors and Ergonomics Society 40 (3) (1998) 469 -477 .

[33] B. Reeves, C. Nass, The Media Equation: How People Treat Computers, Television, and New Media Like Real People and Places, 1st Edition, Cambridge, 1996.

[34] J. Sinclair, P. Hingston, M. Masek, Considerations for the design of exergames, in: GRAPHITE '07: Proceedings of the 5th international conference on Computer graphics and interactive techniques in Australia and Southeast Asia, 2007, pp. 289-295. doi:10.1145/1321261.1321313. URL http: //dx.doi .org/10.1145/1321261.1321313

[35] B. Glaser, A. Strauss, The discovery of grounded theory: Strategies for qualitative research, Aldine, New York, 1967.

[36] F. Mueller, N. Berthouze, Evaluating exertion games experiences from investigating movement-based games, in: R. Bernhaupt (Ed.), Evaluating User Experiences in Games, Springer, Forthcoming.

[37] J. N. Bailenson, N. Yee, Digital chameleons: Automatic assimilation of nonverbal gestures in immersive virtual environments, Psychological Science 16 (10) (2005) 814-819. doi:10.1111/j.1467-9280.2005.01619.x. URL http://dx.doi.org/10.1111/j.1467-9280.2005.01619.x

[38] O. P. John, S. Srivastava, The big-five trait taxonomy: History, measurement, and theoretical perspectives, in: L. Pervin, O. John (Eds.), Handbook of personality: Theory and research, 2nd Edition, Guilford, New York, 1999.

[39] L. G. Pelletier, M. S. Fortier, R. J. Vallerand, K. M. Tuson, N. M. Brière, M. R. Blais, Toward a new measure of intrinsic motivation, extrinsic motivation, and amotivation in sports: The sport motivation scale (sms), Journal of Sport and Exercise Psychology 17 (1995) 35 - 53.

[40] L. Pelletier, R. Vallerand, M. Blais, N. Brière, Leisure motivation scale (lms-28), http://www.er.uqam.ca/nobel/r26710/LRCS/scales/eml28_en.doc (1991).

[41] G. Borg, H. Linderholm, Perceived exertion and pulse rate during graded exercise in various age groups, Acta Med Scand Suppl 472 (1967) 194 206.

[42] M. M. Bradley, P. J. Lang, Measuring emotion: the self-assessment manikin and the semantic differential., J Behav Ther Exp Psychiatry 25 (1) (1994) 49-59.

URL http://view.ncbi.nlm.nih.gov/pubmed/7962581

[43] S. Lindley, J. Le Couteur, N. Bianchi-Berthouze, Stirring up experience through movement in game play: Effects on engagement and social be- haviour, in: SIGCHI conference on Human factors in computing systems, 2008, pp. $511-514$ 